\title{
Rainbow Connection Number and Chromatic Index of Rough Ideal based Rough Edge Cayley Graph
}

\author{
B.Praba $^{1 *}$ and X.A.Benazir Obilia ${ }^{2}$ \\ ${ }^{1}$ Department of Mathematics, SSN College of Engineering, Chennai - 603110, India. \\ ${ }^{2}$ Department of Mathematics, SSN College of Engineering, Chennai - 603110, India. \\ *Corresponding Author E-mail:prabab@ssn.edu.in
}

\begin{abstract}
Rainbow connection number and chromatic index are two significant parameters in the study of graph theory. In this work, rainbow connection number and chromatic index of Rough Ideal based Rough Edge Cayley Graph $G(T(J))$ are evaluated. We prove that the rainbow connection number of $G(T(J))$ is 2 and the chromatic index of $G(T(J))$ is $2\left(2^{n-m}\right)\left(3^{m-1}\right)$.
\end{abstract}

Keywords: Distance, Path, Edge Colouring, Connected

\section{Introduction}

A Computer scientist Z.Pawlak[14] introduced the concept of Rough set theory in the early 1980's as an extension of set theory to deal with the vagueness in the Information system. Colouing of a graph plays an important role in graph theory[1] and it has wide applications. Vizing in his theorem has found the range of the chromatic index for a simple graph. Vizing's theorem and Edge chromatic Graph theory was discussed by Robert Green in 2015 [8]. For interval graphs the rainbow connection number and diameter was obtained by Sudhakaraiah, et.al[11] in 2012.There are various research carried out on algebraic graph theory. Rainbow connection number[10], [12] of Cayley graphs was discussed by Yingbin Ma and Zaiping Lu[13] in 2017. Hengzhe li and Xueliang Li[2] have described the rainbow connection number of graphs with diameter 3 .

As an algebraic approach to Rough set theory the authors Praba.B and R.Mohan[4] defined two operations praba $\Delta$ and $\operatorname{praba} \nabla$ on the set of all rough sets $T$ and proved $T$ to be a rough Lattice. Later the author Praba, et.al[5] proved $T$ to be the Rough semiring under these two operations. The characterization of Rough semiring was given by Manimaran, et.al. The concept of Edge Rough Graph was first introduced by Meilian Liang,et.al[3]. The roughness in Cayley graphs was given by M.H. Shahzamanian, et al[9]. The concept of Rough Ideal based Rough Edge Cayley Graph was introduced by B.Praba and Benazir Obilia[6], [7] as an algebraic graph theoretical approach to Rough set theory. In our earlier work we have obtained the clique number, girth, Wiener index, Maximal independent set and domination number of Rough Ideal based Rough Edge Cayley Graph. This paper is structured as follows: Section 2 gives the prerequisites for the understanding of the article. In section 3 we obtain the diameter and rainbow connection number of Rough Ideal based Rough Edge Cayley Graph. In section 3 we obtain the chromatic index of Rough Ideal based Rough Edge Cayley Graph and section 5 gives the conclusion.

\section{Preliminaries}

\subsection{Graph Theory}

[3] Let $\mathrm{G}$ be a group and $\mathrm{S}$ a set of generators. The Cayley graph $\Gamma(G, S)$ is $(V(\Gamma), E(\Gamma))$ where vertex set $V(\Gamma)$ is $\mathrm{G}$ and the edge set $E(\Gamma)$ consists of all ordered pairs (g,gs) such that $\mathrm{g}$ is in $\mathrm{G}$ and $\mathrm{s}$ is in $\mathrm{S}$. [1] Let $\Gamma$ be a (undirected) graph, and $\mathrm{v}, \mathrm{w}$ two vertices in $V(\Gamma)$. The distance between $\mathrm{v}$ and $\mathrm{w}$, denoted $\mathrm{d}(\mathrm{v}, \mathrm{w})$, is the number of edges in a shortest path connecting them. The diameter of $\Gamma$ is the maximum distance between any two vertices in $V(\Gamma)$. [1] An edge colouring of a graph $G=(V, E)$ is a map $C: E \rightarrow S$, where $\mathrm{S}$ is a set of colours, such that for all $e, f \in E$, if e and $\mathrm{f}$ share a vertex, then $C(e) \neq C(f)$. The chromatic index of a graph $\chi^{\prime}(G)$ is the minimum number of colours needed for a proper colouring of $\mathrm{G}$. [2],[10],[11],[12],[13] A path in an edge-colored graph $\mathrm{G}$ is rainbow if no two edges of the path are colored the same. The rainbow connection number $\mathrm{rc}(\mathrm{G})$ of $\mathrm{G}$ is the smallest integer $\mathrm{k}$ for which there exists a k-edge-coloring of $\mathrm{G}$ such that every pair of distinct vertices of $\mathrm{G}$ is connected by a rainbow path. Vizing's Theorem [8] "For all finite, simple graphs $\mathrm{G}, \Delta(G) \leq \chi^{\prime}(G) \leq \Delta(G)+1$ where $\Delta(G)$ is the maximum degree of a graph."

\subsection{Rough Set Theory}

In this section we consider an approximation space $I=(U, R)$ where $U$ is a non empty finite set of objects, called universal set and $\mathrm{R}$ be an equivalence relation defined on U. [14] For any approximation space, the equivalence classes induced by $R$ is defined by $[x]=\{y \in U \mid(x, y) \in R\}$. For any $X \subseteq U$, the lower approximation is defined as $R_{-}(X)=\{x \in U \mid[x] \subseteq X\}$ and the upper approximation is defined by $R^{-}(X)=\{x \in U \mid[x] \cap X=\phi\}$. The rough set corresponding to $X$ is $R S(X)=\left(R_{-}(X), R^{-}(X)\right)$. [4] 
If $X \subseteq U$, then the number of equivalence classes(Induced by $R$ ) contained in $X$ is called as the Ind.weight of $X$. It is denoted by $I_{W}(X)$. [4] Let $X, Y \subseteq U$. The Praba $\Delta$ is defined as $X \Delta Y=$ $X \cup Y$, if $I_{W}(X \cup Y)=I_{W}(X)+I_{W}(Y)-I_{W}(X \cap Y)$. If $I_{W}(X \cup Y)>$ $I_{W}(X)+I_{W}(Y)-I_{W}(X \cap Y)$, then identify the equivalence class obtained by the union of $X$ and Y. Then delete the elements of that class belonging to $\mathrm{Y}$. Call the new set as Y. Now obtain $X \Delta Y$. Repeat the process until $I_{W}(X \cup Y)=I_{W}(X)+I_{W}(Y)-I_{W}(X \cap Y)$. [4] Praba $\nabla$ of $X$ and $Y$ is denoted by $X \nabla Y$ and it is defined as $X \nabla Y=\{x \mid[x] \subseteq X \cap Y\} \cup P_{X \cap Y}$, where $X, Y \subseteq U$ and $P_{X \cap Y}$ contains those elements of $U$ whose corresponding equivalence classes are not a subset of $X \cap Y$ but will have non empty intersection with $\mathrm{X}$ and $\mathrm{Y}$.

Theorem 2.1. [5] For any approximation space $I=(U, R),(T, \Delta, \nabla)$ is a semiring called the Rough semiring.

Remarks 2.1. [5] Without loss of generality let us assume that there are n-equivalence classes $\left\{X_{1}, X_{2}, \ldots, X_{n}\right\}$ in $U$. Out of which there are $m$ classes $\left\{X_{1}, X_{2}, \ldots, X_{m}\right\}$ (say) have cardinalities greater than one and the remaining $n-m$ clsses have cardinality equal to one. Note that $|T|=2^{n-m} 3^{m}$. Let $B=\left\{x_{i} \mid x_{i} \in X_{i}, i=1,2, \ldots, m\right\}$ and $J=\{R S(X) \mid X \in P(B)\}$ then $J$ is an ideal of the Rough semiring called the Rough Ideal .

\subsection{Rough Ideal based Rough Edge Cayley Graph}

[6],[7] In this section, we consider an approximation space $I=(U, R)$ where $U$ is the non empty finite set of objects and $R$ is an equivalence relation on $U$. Let $(T, \Delta, \nabla)$ be the rough semiring induced by $I$. Let $B$ be the set of representative elements of $X_{i}, i=1,2, \ldots, m$ and $J$ be the rough ideal as in the previous section. We also assume that $M$ is the union of none, one or more equivalence classes whose cardinality is equal to one and $M^{\prime}$ is the union of one or more equivalence classes whose cardinality is equal to one.

[6],[7] Rough Ideal based Rough Edge Cayley Graph denoted $G(T(J))=(V, E)$ where $V \in T$ and

$E=\{(R S(X), R S(Z)) \mid R S(X) \nabla R S(Y)=R S(Z), R S(Y) \in J\}$

\subsection{Category Graph}

[6],[7] Cardinality of $T(|T|)$ is $2^{n-m} 3^{m}$. We divide the vertices (the elements of $T$ ) into 13 categories in such a way that all elements belonging to a particular category will behave similarly. The vertices are grouped in categories by the following conditions:

1. The degree of each vertex in a particular category will be same

2. The distances from any vertex of a particular category to elements of other categories will be same.

In Table: 1 , we give the 13 categories and degree of the vertices in each category.

[6],[7] The category graph corresponding to a given Rough Ideal based Rough Edge Cayley graph is defined as follows: The vertices of the category graph $(C G)$ are $C_{1}, C_{2}, \ldots, C_{13}$. Two vertices $C_{i}$ and $C_{j}$ are connected if elements in $C_{i}$ are connected to elements in $C_{j}$ by an edge in the Rough Ideal based Rough Edge Cayley graph. The Category graph is shown in Figure: 1
Table 1: Categories and Degrees of each vertex in the categories

\begin{tabular}{|c|c|c|}
\hline Category $\left(C_{i}\right)$ & Vertices & $\begin{array}{c}\text { Degree of each vertex } \\
\text { in the Category } d\left(C_{i}\right)\end{array}$ \\
\hline$C_{1}$ & $R S(\phi)$ & $2^{n-m}\left(3^{m}-2^{m}\right)-1$ \\
\hline$C_{2}$ & $R S\left\{x_{i}\right\}$ & $2\left(2^{n-m}\right)\left(3^{m-1}\right)$ \\
\hline$C_{3}$ & $R S\left(X_{i} \cup M\right)$ & 2 \\
\hline$C_{4}$ & $M^{\prime}$ & 1 \\
\hline$C_{5}$ & $R S\left(\left\{x_{1} x_{2}, \ldots, x_{r}\right\}\right)$ & $2^{r}(3)^{m-r}(2)^{n-m}+2^{r}-2$ \\
\hline$C_{6}$ & $R S\left(\left\{x_{1} x_{2}, \ldots, x_{r}\right\} \cup M^{\prime}\right)$ & $2^{r}$ \\
\hline$C_{7}$ & $R S\left(\left\{X_{1} X_{2}, \ldots, X_{r}\right\} \cup M\right)$ & $2^{r}$ \\
\hline$C_{8}$ & $R S\left(\left\{x_{1} x_{2}, \ldots, x_{m}\right\}\right)$ & $2^{m}+2^{n}-3$ \\
\hline$C_{9}$ & $R S\left(\left\{x_{1} x_{2}, \ldots, x_{m}\right\} \cup M^{\prime}\right)$ & $2^{m}-1$ \\
\hline$C_{10}$ & $R S\left(\left\{X_{1} X_{2}, \ldots, X_{m}\right\} \cup M\right)$ & $2^{m}-1$ \\
\hline$C_{11}$ & $R S\left(Q_{r} \cup M\right)$ & $2^{r}$ \\
\hline$C_{12}$ & $R S\left(Q_{m} \cup M\right)$ & $2^{m}-1$ \\
\hline$C_{13}$ & $R S\left(\left\{x_{i}\right\} \cup M^{\prime}\right)$ & 2 \\
\hline & & \\
\hline
\end{tabular}

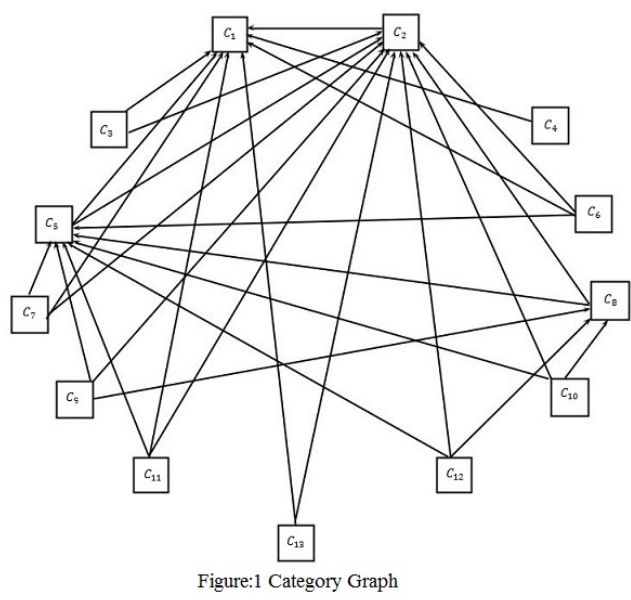

\section{Rainbow Connection Number of the Rough Ideal based Rough Edge Cayley graph}

Throughout this paper, we consider the Rough Ideal based Rough Edge Cayley graph $G(T(J))$ with the corresponding category graph $C G$. Note that for any category $C_{i}$, all the vertices in $C_{i}$ will have the same degree in $G(T(J))$ and hence the degree of the vertices in $C_{i}$ is denoted by $d\left(C_{i}\right)$

In this section we are going to obtain the diameter of $G(T(J))$ in two different cases namely when all the equivalence classes have cardinality greater than one and if there exists one equivalence class of cardinality greater than one. Also, the Rainbow connection number of $G(T(J))$ is obtained..

Theorem 3.1. Diameter of $G(T(J))$ is 2 or 3 .

Proof. Case: 1 When $n=m$

Consider the Categories $C_{1}$ and $C_{2}$ from the Category graph corresponding to $G(T(J))$. The category $C_{1}$ consists of the single element $R S(\phi)$ and $C_{2}$ consists of the elements of the form $R S\left(\left\{x_{i}\right\}\right), x_{i} \in B . \quad R S\left(\left\{x_{i}\right\}\right)$ is adjacent to all the vertice of the form $R S(Y)$ such that $R S\left(\left\{x_{i}\right\}\right) \nabla R S(X)=R S(Y)$ where $R S(X) \in J$ and $R S(Y) \in T$ and $R S(X) \nabla R S(Y)=R S\left(\left\{x_{i}\right\}\right)$. Elements of the form $R S\left(X_{i}\right), R S\left(\left\{x_{1}, x_{2}, \ldots, x_{r}\right\}\right), R S\left(\left\{x_{1}, x_{2}, \ldots, x_{r}\right\} \cup\right.$ $\left.M^{\prime}\right), R S\left(\left\{X_{1}, X_{2}, \ldots, X_{r}\right\} \cup M\right), R S\left(\left\{x_{1}, x_{2}, \ldots, x_{m}\right\}\right), R S\left(\left\{x_{1}, x_{2}, \ldots, x_{m}\right\} \cup\right.$ $\left.M^{\prime}\right), R S\left(\left\{X_{1}, X_{2}, \ldots, X_{m}\right\} \cup M\right), R S\left(Q_{r} \cup M\right), R S\left(Q_{m} \cup M\right)$ and $R S\left(X_{i} \cup M^{\prime}\right)$ are adjacent to $R S\left(\left\{x_{i}\right\}\right)$. (i.e.) The category $C_{2}$ is adjacent to the categories $C_{3}, C_{5}, C_{6}, C_{7}, C_{8}, C_{9}, C_{10}, C_{11}, C_{12}$ and $C_{13}$. By the similar argument, the category $C_{1}$ is adjacent to the categories $C_{3}, C_{5}, C_{6}, C_{7}, C_{11}$ and $C_{13}$. The categories $C_{8}, C_{9}, C_{10}, C_{12}$ are adjacnt only to $C_{2}$ and not to $C_{1}$. Hence the 
categories $C_{8}, C_{9}, C_{10}, C_{12}$ are at a distance 1 to $C_{2}$ and are at a distance 2 to $C_{1}$. The category $C_{4}$ will have no elements in thi case. Thereby we have exhausted all the categories. Hence the diameter of $G(T(J))$ is 2 .

Case: 2 When $m<n$

By the similar argument in Case:1, the categories $C_{3}, C_{5}, C_{6}, C_{7}, C_{8}, C_{9}, C_{10}, C_{11}, C_{12}$ and $C_{13}$ are adjacent to the category $C_{2}$ and the category $C_{1}$ is adjacent to the categories $C_{3}, C_{5}, C_{6}, C_{7}, C_{11}$ and $C_{13}$. The categories $C_{8}, C_{9}, C_{10}, C_{12}$ are adjacnt only to $C_{2}$ and not to $C_{1}$. The category $C_{4}$ is adjacent only to $C_{1}$. Hence the categories $C_{8}, C_{9}, C_{10}, C_{12}$ are at a distance 1 to $C_{2}$, at a distance 2 to $C_{1}$ and at a distance 3 to $C_{4}$. Hence the diameter of $G(T(J))$ is 3 .

Corollary 3.1. Rainbow connection number of $G(T(J))$ is 2 .

Proof. It is trivial by the theorem 3.1.

Example 3.1. Let $u$ s consider the approximation space $I=$ $(U, R)$ where $U=\left\{x_{1}, x_{2}, x_{3}, x_{4}\right\}$. Let $X_{1}=\left[x_{1}\right]=\left\{x_{1}, x_{3}\right\}$ and $X_{2}=\left[x_{2}\right]=\left\{x_{2}, x_{4}\right\}$ be the equivalence classes induced by $R$. $T=\left\{R S(\phi), R S(U), R S\left(X_{1}\right), R S\left(X_{2}\right), R S\left(\left\{x_{1}\right\}\right), R S\left(\left\{x_{2}\right\}\right), R S\left(X_{1} \cup\right.\right.$ $\left.\left.\left\{x_{2}\right\}\right), R S\left(\left\{x_{1}\right\} \cup X_{2}\right), R S\left(\left\{x_{1}\right\} \cup\left\{x_{2}\right\}\right)\right\}$.

The vertices of $G(T(J))$ are the elements of $T$. Each element $R S(X)$ in $T$ is connected to $R S(X) \nabla R S(Y)$ where $R S(Y) \in$ $J$. If $B=\left\{x_{1}, x_{2}\right\}$ then $P(B)=\left\{\phi,\left\{x_{1}\right\},\left\{x_{2}\right\},\left\{x_{1}, x_{2}\right\}\right\}, J=$ $\left\{R S(\phi), R S\left(\left\{x_{1}\right\}\right), R S\left(\left\{x_{2}\right\}\right), R S\left(\left\{x_{1}, x_{2}\right\}\right)\right\}$

There are 2 equivalence classes and those 2 are of cardinality greater than one. (i.e.) $n=2$ and $m=2$. For example, consider the categories $C_{1}$ and $C_{3}$. The only element in category $C_{1}$ is $R S(\phi)$. The elements of the category $C_{3}$ are $R S\left(X_{1}\right)$ and $R S\left(X_{2}\right) . R S\left(X_{1}\right) \nabla R S\left(\left\{x_{2}\right\}\right)=R S(\phi)$ where $R S\left(\left\{x_{2}\right\}\right) \in$ $J$. $R S\left(X_{2}\right) \nabla R S\left(\left\{x_{1}\right\}\right)=R S(\phi)$ where $R S\left(\left\{x_{1}\right\}\right) \in J$. Hence, the elements of the category $C_{3}$ are connected to $C_{1}$. Consider the categories $C_{8}$ and $C_{12}$ The only element in category $C_{8}$ is $R S\left(\left\{x_{1}, x_{2}\right\}\right) . R S\left(\left\{x_{1}\right\} \cup X_{2}\right) \nabla R S\left(\left\{x_{1}, x_{2}\right\}\right)=R S\left(\left\{x_{1}, x_{2}\right\}\right)$, $R S\left(X_{1} \cup\left\{x_{2}\right\}\right) \nabla R S\left(\left\{x_{1}, x_{2}\right\}\right)=R S\left(\left\{x_{1}, x_{2}\right\}\right)$. Hence the elements of $C_{12}$ are connected to $C_{8}$. By using the similar argument between all categories, the category graph is obtained and the category graph in this case when $n=m=2$ is shown in figure:2. By case $: 1$ of theorem 3.1, the diameter of $G(T(J)$ is 2 and the rainbow conncetion number is also 2.

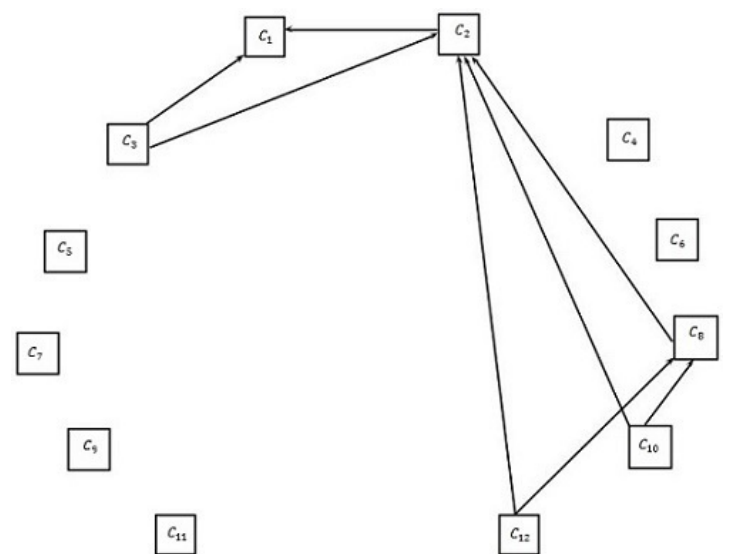

Figure:2 Category graph for $\mathrm{n}=2$ and $\mathrm{m}=2$
Example 3.2. Let $I=(U, R)$ be an approximation space,then $U=\left\{x_{1}, x_{2}, x_{3}, x_{4}, x_{5}, x_{6}\right\}$. Let $\left\{X_{1}, X_{2}, X_{3}\right\}$ be the equivalence classes induced by $R$. $X_{1}=\left\{x_{1}, x_{3}\right\}=\left[x_{1}\right], X_{2}=\left\{x_{2}, x_{4}, x_{6}\right\}=\left[x_{2}\right]$ and $X_{3}=\left\{x_{5}\right\}=\left[x_{5}\right] .\left\{X_{1}, X_{2}\right\}$ are the equivalence classes whose cardinality is greater than one and $\left\{x_{1}, x_{2}\right\}$ be the pivot elements of these classes. $\left\{X_{3}\right\}$ is the equivalence classes whose cardinality is equal to one. In this example,

$T=\left\{R S(\phi), R S\left(\left\{x_{1}\right\}\right), R S\left(\left\{x_{2}\right\}\right), R S\left(X_{1}\right), R S\left(X_{2}\right), R S\left(X_{3}\right), R S\left(X_{1} \cup\right.\right.$ $\left.\left\{x_{2}\right\}\right), R S\left(\left\{x_{1}\right\} \cup X_{2}\right), R S\left(\left\{x_{1}\right\} \cup\left\{x_{2}\right\}\right), R S\left(\left\{x_{1}\right\} \cup X_{3}\right), R S\left(\left\{x_{2}\right\} \cup\right.$ $\left.X_{3}\right), R S\left(X_{1} \cup X_{3}\right), R S\left(X_{2} \cup X_{3}\right), R S\left(\left\{x_{1}\right\} \cup X_{2} \cup X_{3}\right), R S\left(X_{1} \cup\left\{x_{2}\right\} \cup\right.$ $\left.\left.X_{3}\right), R S\left(\left\{x_{1}\right\} \cup\left\{x_{2}\right\} \cup X_{3}\right), R S(U)\right\}$ $B=\left\{x_{1}, x_{2}\right\}$ then $J=\left\{R S(\phi), R S\left(\left\{x_{1}\right\}\right), R S\left(\left\{x_{2}\right\}\right), R S\left(\left\{x_{1}, x_{2}\right\}\right)\right\}$ In this example, there are 3 equivalence classes and out of which the cardinalities of 2 equivalence classes are greater than one. (i.e) $n=3$ and $m=2$. The Category Graph of $G(T(J))$ is shown in figure:3.

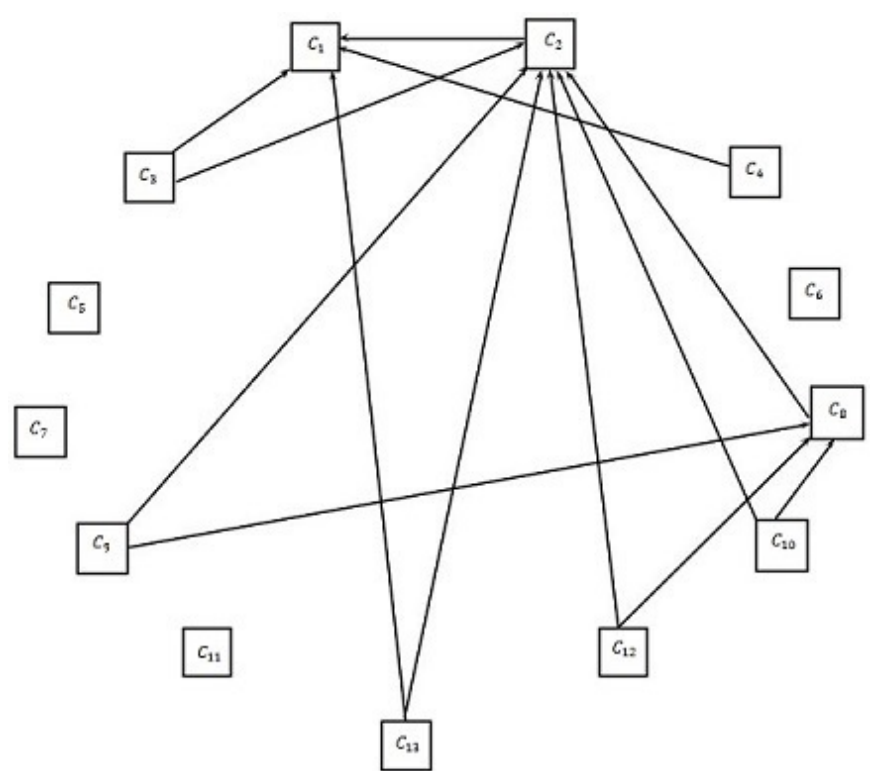

Figure: 3 Category graph for $\mathrm{n}=3$ and $\mathrm{m}=2$

By case :2 of theorem 3.1, the diameter of $G(T(J)$ is 3 and the rainbow conncetion number is 2 .

\section{Chromatic Index of the Rough Ideal based Rough Edge Cayley Graph}

Theorem 4.1. Chromatic Index of $G(T(J))$ is $2\left(2^{n-m}\right)\left(3^{m-1}\right)$.

Proof. To prove this, first we prove that the vertices of $C_{2}$ are having the highest degree in $G(T(J))$. We prove this comparing the degree of the vertices in each of the categories. Consider the degrees of each categories in the category graph corresponding to $G(T(J))$.

$d\left(C_{1}\right)=\left(2^{n-m}\right)\left(3^{m}-2^{m}\right)-1$

$d\left(C_{2}\right)=2\left(2^{n-m}\right)\left(3^{m-1}\right)$

Hence $d\left(C_{2}\right)>d\left(C_{1}\right)$.

$d\left(C_{3}\right), d\left(C_{13}\right)=2$ 
It is obvious that $d\left(C_{3}\right), d\left(C_{13}\right)<d\left(C_{2}\right)$.

$d\left(C_{6}\right), d\left(C_{7}\right), d\left(C_{11}\right)=2^{r}, 1<r<m$.

Maximum degree these $d\left(C_{6}\right), d\left(C_{7}\right), d\left(C_{11}\right)$ can have is $2^{m-1}$. Hence $d\left(C_{2}\right)>d\left(C_{6}\right), d\left(C_{2}\right)>d\left(C_{7}\right)$ and $d\left(C_{2}\right)>d\left(C_{11}\right)$

$d\left(C_{9}\right), d\left(C_{10}\right), d\left(C_{12}\right)=2^{m}-1$.

Hence $d\left(C_{2}\right)>d\left(C_{9}\right), d\left(C_{2}\right)>d\left(C_{10}\right), d\left(C_{2}\right)>d\left(C_{12}\right)$.

$d\left(C_{4}\right)=1<d\left(C_{2}\right)$

$d\left(C_{8}\right)=2^{m}+2^{n}-3<d\left(C_{2}\right)$

From equation (1) to (7) we have exhausted all the degrees of all the categories except $d\left(C_{5}\right)$.

Now consider $d\left(C_{5}\right)=2^{r}(3)^{m-r}(2)^{n-m}+2^{r}-2,1<r<m$.

$\operatorname{Max}\left\{d\left(C_{5}\right)\right\}=3\left(2^{n-1}\right)+2^{m-1}-2$.

To prove $d\left(C_{2}\right)>d\left(C_{5}\right)$

(i.e.) to prove for any $n \geq 3, m \geq 3,3\left(2^{n-1}\right)+2^{m-1}-$ $2<2\left(2^{n-m}\right)\left(3^{m-1}\right)$.

(i.e.) to prove for any $n \geq 3, m \geq 3$,

$3\left(2^{n-2}\right)+2^{m-2}-1<\left(2^{n-m}\right)\left(3^{m-1}\right.$

This can be done by induction on $n$ and $m$.

When $m=3$

It is obvious that L.H.S $<$ R.H.S

Induction on $n$ : When $n=3$

Obviously L.H.S $<$ R.H.S.

Assume for any $n=k$,

$3\left(2^{k-2}\right)+1<3^{2}\left(2^{k-3}\right)$

To prove that for any $n=k+1,3\left(2^{k-1}\right)+1<3^{2}\left(2^{k-2}\right)$

$$
\begin{aligned}
\text { R.H.S } & =3^{2}\left(2^{k-2}\right) \\
& =2\left(3^{2}\right)\left(2^{k-3}\right) \\
& >2\left(3\left(2^{k-2}\right)+1\right) \quad(\text { by }(9)) \\
& >(3)\left(2^{k-1}\right)+1=\text { L.H.S }
\end{aligned}
$$

Hence for $m=3$ equation (8) is true.

Assume that for any $m=p$,

$3\left(2^{n-2}\right)+2^{p-2}-1<\left(2^{n-p}\right)\left(3^{p-1}\right)$

To prove for any $m=p+1,3\left(2^{n-2}\right)+2^{p-1}-1<\left(2^{n-p-1}\right)\left(3^{p}\right)$ Induction on $n$ :

When $n=p+1$, L.H.S $=2^{p+1}-1$

R.H.S $=3^{p}$.

Hence L.H.S $<$ R.H.S.

Assume that for any $n=l>p+1$,

$$
3\left(2^{l-2}\right)+2^{p-1}-1<\left(2^{l-p-1}\right)\left(3^{p}\right)
$$

To prove for any $n=l+1,3\left(2^{l-1}\right)+2^{p-1}-1<\left(2^{l-p}\right)\left(3^{p}\right)$

$$
\begin{aligned}
\text { R.H.S } & =\left(2^{l-p}\right)\left(3^{p}\right) \\
& =2\left(2^{l-p-1}\right)\left(3^{p}\right) \\
& >2\left(3\left(2^{l-2}\right)+2^{p-1}-1\right) \quad(\text { by }(11)) \\
& >3\left(2^{l-1}\right)+2^{p}-2 \\
& >3\left(2^{l-1}\right)+2^{p-1}-1=\text { L.H.S }
\end{aligned}
$$

Hence for any $n \geq 3, m \geq 3,3\left(2^{n-2}\right)+2^{m-2}-1<\left(2^{n-m}\right)\left(3^{m-1}\right)$. (i.e) $d\left(C_{2}\right)>d\left(C_{5}\right)$ and hence $d\left(C_{2}\right)$ is maximum. Hence by Vizing's theorem, the chromatic index of $G(T(J))$ is $2\left(2^{n-m}\right)\left(3^{m-1}\right)$
Remarks 4.1. In the above theorem, we have proved that $d\left(C_{2}\right)$ is maximum. (i.e.) $\Delta(G(T(J)))=d\left(C_{2}\right)$. By Vizing's theorem, the chromatic index is $\Delta(G(T(J)))$ or $\Delta(G(T(J)))+1$. We claim that the chromatic index of $G(T(J))$ is $d\left(C_{2}\right)$ and the proof of the fact that the chromatic index of $G(T(J))$ is not $d\left(C_{2}\right)+1$ is left as an open problem.

Example 4.1. For example 3.1, the categories of vertices and their corresponding degrees are shown in table : 2

From table $: 2$, we observe that $d\left(C_{2}\right)=6$ and its maximum. Hence

Table 2: Categories of vertices with its degrees when $n=2$ and $m=2$.

\begin{tabular}{|c|c|c|}
\hline Categories & Vertices & Degrees $d\left(C_{i}\right)$ \\
\hline$C_{1}$ & $R S(\phi)$ & 4 \\
\hline$C_{2}$ & $R S\left(\left\{x_{1}\right\}\right), R S\left(\left\{x_{2}\right\}\right)$ & 6 \\
\hline$C_{3}$ & $R S\left(\left\{X_{1}\right\}\right), R S\left(\left\{X_{2}\right\}\right)$ & 2 \\
\hline$C_{4}$ & - & - \\
\hline$C_{5}$ & - & - \\
\hline$C_{6}$ & - & - \\
\hline$C_{7}$ & - & - \\
\hline$C_{8}$ & $R S\left(\left\{x_{1}, x_{2}\right\}\right)$ & 5 \\
\hline$C_{9}$ & - & - \\
\hline$C_{10}$ & $\mathrm{RS}(\mathrm{U})$ & 3 \\
\hline$C_{11}$ & - & - \\
\hline$C_{12}$ & $R S\left(\left\{x_{1}\right\} \cup X_{2}\right), R S\left(X_{1} \cup\left\{x_{2}\right\}\right)$ & 3 \\
\hline$C_{13}$ & - & - \\
\hline
\end{tabular}

by theorem 4.1, the chromatic index of $G(T(J))$ is 6 . The diagramatical justification is shown in figure :4.

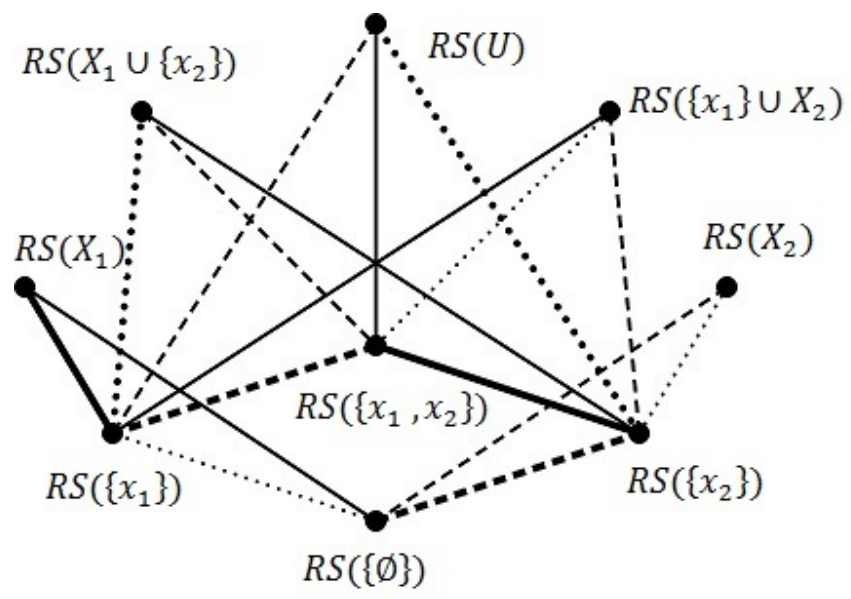

Figure :4 Edge Colouring of $G(T(J))$ when $\mathrm{n}=2$ and $\mathrm{m}=2$

\section{Conclusion}

In this work, the rainbow conncetion number of $G(T(J)$ is 2 and the chromatic index of $\mathrm{G}(\mathrm{T}(\mathrm{J}))$ is $2\left(2^{n-m}\right)\left(3^{m-1}\right)$ has been proved and illustrated with examples. As we have used Vizing's theorem, the proof of the statement that the chromatic index of $G(T(J)$ is not $2\left(2^{n-m}\right)\left(3^{m-1}\right)+1$ is left as an open prroblem.

\section{Acknowledgement}

The authors thank the Management and the Principal, SSN Institutions for the completion of this paper and providing encouragement and support to carry out this research. 


\section{References}

[1] J.A.Bondy and U.S.R.Murty, (1976), Graph theory with applications, University of Waterloo, Ontario, Canada.

[2] Hengzhe li, Xueliang li, (2017), rainbow Connection Number of Graphs with Diameter 3, Discussiones Mathematicae, No:37, Pg: 141-154.

[3] Meilian Liang, Binmei Liang, (2011), Linna Wei and Xiadong Xu, Edge rough graph and its applications, 8th International conference on Fuzzy systems and Knowledge discovery, Pg:335-338.

[4] B. Praba and R. Mohan, (2013), Rough Lattice, International Journal of Fuzzy Mathematics and Systems, Volume 3, Number 2, pp. 135151

[5] B.Praba, V.M.Chandrasekaran and A.Maninmaran, (2015), Semiring on Rough sets, Indian Journal of Science and Technology, Vol.8(3), 280-286,

[6] B.Praba and Benazir obilia.X.A, Domination number of Rough Ideal based rough Edge Cayley Graph,Accepted for Cambridge summit 2018.

[7] B.Praba and Benazir obilia.X.A,, (2017), Domination number of Rough Ideal based rough Edge Cayley Graph through Category Graph, International Journal of Pure and Applied Mathematics, No. 9, 187-191.

[8] Robert Green, Vizing's Theorem and Edge Chromatic Graph Theory, math.uchicago.edu/ may/REU2015/REUPapers/Green.pdf.

[9] M.H. Shahzamanian,M. Shirmohammadi, B. Davvaz, (2010), Roughness in Cayley Graphs, InformationSciences 180, 3362-3372.

[10] L. Sunil Chandran, Anita Das, Deepak Rajendraprasad, and Nithin M. Varma, , (2010), (Rainbow Connection Number and Connected Dominating Sets, arXiv : 1010.2296v1 [math.CO].

[11] Dr. A. Sudhakaraiah, E.Gnana Deepika, V. Rama Latha , (2012), Rainbow Connection Number and the Diameter of Interval Graphs, IOSR Journal of Mathematics (IOSRJM) Volume 1, Issue 1, PP 36-43.

[12] Xueliang Li , Sujuan Liu, (2012), Rainbow Connection Number and Connectivity, The electronic Journal of Combiatorics, 19.

[13] Yingbin Ma, Zaiping Lu, (2017), Rainbow connection numbers of Cayley graphs, Journal of Combinatorial Optimization, No:34, pg:182-19.

[14] Zdzislaw Pawlak, "Rough Sets", (1982), International Journal of Computer and Information Sciences, Vol.11, 341-356. 